Michalik, Kerstin

\title{
Ungewissheit als Herausforderung und Chance - Perspektiven von Lehrerinnen und Kindern auf das philosophische Gespräch
}

Philosophieren mit Kindern - Forschungszugänge und -perspektiven. Opladen; Berlin; Toronto : Verlag Barbara Budrich 2018, S. 175-187

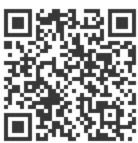

Quellenangabe/ Reference:

Michalik, Kerstin: Ungewissheit als Herausforderung und Chance - Perspektiven von Lehrerinnen und Kindern auf das philosophische Gespräch - In: Philosophieren mit Kindern - Forschungszugänge und -perspektiven. Opladen; Berlin; Toronto : Verlag Barbara Budrich 2018, S. 175-187 - URN: urn:nbn:de:0111-pedocs-207715 - DOI: 10.25656/01:20771

https://nbn-resolving.org/urn:nbn:de:0111-pedocs-207715

https://doi.org/10.25656/01:20771

in Kooperation mit / in cooperation with:

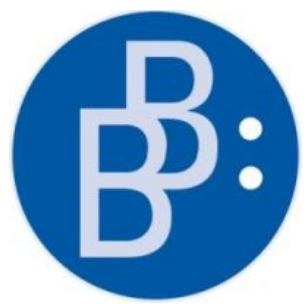

https://www.budrich.de

\section{Nutzungsbedingungen}

Dieses Dokument steht unter folgender Creative Commons-Lizenz: http://creativecommons.org/licenses/by-sa/4.0/deed.de - Sie dürfen das Werk bzw. den Inhalt vervielfältigen, verbreiten und öffentlich zugänglich machen sowie Abwandlungen und Bearbeitungen des Werkes bzw. Inhaltes anfertigen, solange sie den Namen des Autors/Rechteinhabers in der von inm festgelegten Weise nennen und die daraufhin neu entstandenen Werke bzw. Inhalte nur unter Verwendung von Lizenzbedingungen weitergeben, die mit denen dieses Lizenzvertrags identisch, vergleichbar oder kompatibel sind.

Mit der Verwendung dieses Dokuments erkennen Sie die Nutzungsbedingungen an.

\section{Terms of use}

This document is published under following Creative Commons-License: http://creativecommons.org/licenses/by-sa/4.0/deed.en - You may copy distribute and transmit, adapt or exhibit the work or its contents in public and alter, transform, or change this work as long as you attribute the work in the manner specified by the author or licensor. New resulting works or contents must be distributed pursuant to this license or an identical or comparable license.

By using this particular document, you accept the above-stated conditions of use.

\section{Kontakt / Contact:}

\section{peDOCS}

DIPF | Leibniz-Institut für Bildungsforschung und Bildungsinformation

Informationszentrum (IZ) Bildung

E-Mail: pedocs@dipf.de

Internet: www.pedocs.de

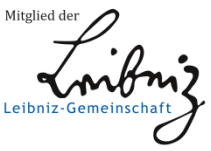


Kerstin Michalik

Ungewissheit als Herausforderung und Chance Perspektiven von Lehrerinnen und Kindern auf das philosophische Gespräch

Verlag Barbara Budrich

Opladen • Berlin • Toronto 2018 
Der Aufsatz Ungewissheit als Herausforderung und ChancePerspektiven von Lehrerinnen und Kindern auf das

philosophische Gespräch von Kerstin Michalik steht unter der Creative Commons Lizenz Attribution-ShareAlike 4.0 International (CC BY-SA 4.0): https://creativecommons.org/licenses/by-sa/4.0/

Diese Lizenz erlaubt die Verbreitung, Speicherung, Vervielfältigung und Bearbeitung bei Verwendung der gleichen CC-BY-SA 4.0-Lizenz und unter Angabe der UrheberInnen, Rechte, Änderungen und verwendeten Lizenz.

Der Aufsatz ist erschienen in:

Michalik, Kerstin/de Boer, Heike (Hrsg.) (2018): Philosophieren mit Kindern - Forschungszugänge und -perspektiven. Opladen: Verlag Barbara Budrich, S. 13-32.

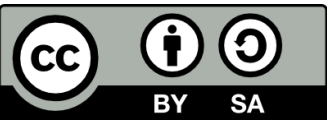

Dieser Beitrag steht im Open-Access-Bereich der Verlagsseite zum kostenlosen Download bereit (https://doi.org/10.3224/84742105.13).

ISBN 978-3-8474-2105-4

DOI $\quad 10.3224 / 84742105.13$ 


\section{Ungewissheit als Herausforderung und Chance - Perspektiven von Lehrerinnen und Kindern auf das philosophische Gespräch}

\section{Kerstin Michalik}

Auf der Basis von Interviews mit Grundschullehrkräften und Gruppendiskussionen mit Grundschulkindern werden Erfahrungen von Lehrkräften und Kindern mit dem Philosophieren mit Kindern auf der Basis inhaltsanalytischen Verfahren rekonstruiert. Die Ergebnisse zeigen, dass regelmäßiges Philosophieren mit Kindern Auswirkungen auf das pädagogische Selbstverständnis, das unterrichtliche Handeln und auch die Persönlichkeitsentwicklung von Lehrkräften haben können. Im Hinblick auf die Kinder wird deutlich, dass es neben den Inhalten des philosophischen Gespräches vor allem der offene Prozess des gemeinsamen Nachdenkens ist, der für sie wichtig und bedeutsam ist. Dabei erweist sich das für das philosophische Gespräch konstitutive Merkmal der „Ungewissheit“ als eine wichtige Komponente im gemeinsamen Deutungshorizont.

\section{Einleitung}

Welchen Sinn sehen die Lehrerinnen im Philosophieren? Welche Veränderungen und Entwicklungen nehmen sie bei den Kindern und auch sich selbst wahr? Mit welchen Problemen und Herausforderungen sehen sie sich konfrontiert? Was ist den Kindern am Philosophieren besonders wichtig und welche Unterschiede sehen sie gegenüber anderen Lern- und Unterrichtssituationen? Die Untersuchung der subjektiven Perspektiven von Lehrkräften und Kindern, ihrer Wahrnehmungen, Bedeutungszuschreibungen und Bewertungen, ist ein Forschungszugang, der nicht von außen mit dem Anspruch der Objektivität auf das Geschehen blickt, sondern die Erfahrungen der Beteiligten zu erfassen sucht. Die Rekonstruktion der Bedeutung des gemeinsamen Philosophierens aus der Sicht der Akteure - der Lehrkräfte und der Kinder - stellt eine wichtige Ergänzung zur bisherigen, überwiegend quantitativ ausgerichteten Wirkungsforschung zum Philosophieren mit Kindern (siehe Michalik/Wirkungen in diesem Band) dar.

Mit diesem Untersuchungsschwerpunkt, der in der Forschung erst seit den letzten Jahren verstärkt Berücksichtigung findet (vgl. Barrow 2015; Scholl 2014; Scholl/Nichols/Burgh 2014; 2016, Topping 2007; Gorard/Sidddiqui/See 2015; Siddiqui/Gorard/See 2017), werden Aspekte und Facetten des 
Philosophierens mit Kindern sichtbar, die mit standardisierten quantitativen Verfahren nicht oder nur ansatzweise erfassbar sind. Die Ergebnisse sind sowohl für die praxisbezogene Frage nach Gelingensbedingungen des Philosophierens im Rahmen von Schule und Unterricht als auch im Hinblick auf Fragestellungen der Professionalisierungsforschung und der Kindheitsforschung relevant. Die Rekonstruktion des Philosophierens aus der Perspektive der Kinder ist zudem ein Forschungszugang, der den Kindern als zentrale Figuren des Geschehens eine eigene Stimme gibt, ihre Wahrnehmungen, Einschätzungen und Bewertungen ernst nimmt und damit den Grundprinzipien des Ansatzes des Philosophierens mit Kindern in besonderem Maße entspricht.

\section{Methodische Anmerkung}

In der vorliegenden Untersuchung erfolgte die Datenerhebung zu den Lehrkräften in Form von halbstrukturierten, leitfadengestützten Interviews, da es einerseits darum ging, bestimmte Aspekte des Philosophierens mit Kindern näher zu beleuchten, andererseits hinreichend Offenheit für eigene Themen und Relevanzsetzungen der Lehrkräfte vorhanden sein sollte (vgl. Flick 2016: 203f.). Die Daten stammen aus zwei Erhebungen (Michalik 201; Haller 2015) ${ }^{1}$, in deren Rahmen jeweils fünf und sechs, also insgesamt elf Lehrkräfte überwiegend aus Hamburg, im Rahmen von 20-40-minütigen Gesprächen interviewt wurden. Die transkribierten Tonaufnahmen wurden auf der Grundlage der qualitativen Inhaltsanalyse nach Mayring (2015) im Rahmen eines induktiven Vorgehens kodiert und kategorisiert, um Inhalte und Themenschwerpunkte herauszuarbeiten. Bei den Lehrkräften handelte es sich um Grundschullehrerinnen, Sonderpädagoginnen und Sozialpädagoginnen im Alter von 28 bis 66 Jahren, die teils im Rahmen des Philosophierens als Unterrichtsprinzips (in den Fächern Kunst, Religion, Mathematik, Deutsch und Sachunterricht), teils im Rahmen von Ganztags- oder Wahlangeboten oder auch im Förderunterricht Erfahrungen mit dem Philosophieren mit Kindern gesammelt hatten.

Parallel zu den Interviews mit den Lehrkräften fanden Gruppendiskussionen mit insgesamt 70 Grundschulkindern aus drei verschiedenen jahrgangsübergreifenden Lerngruppen (Klasse 1-3, d.h. 6-9 Jahre, und Klasse 4-6, d.h. 10-12 Jahre) einer Hamburger Grundschule statt (Michalik 2012). In allen drei jahrgangsübergreifenden Lerngruppen haben die Lehrerinnen zuvor zwischen sechs Monaten und zwei Jahren regelmäßig mit der gesamten Lerngruppe philosophiert. Die insgesamt fünf Gruppendiskussionen erfolgten in Gruppen von jeweils 10-12 Kindern. Die Gespräche wurden videografiert, transkribiert und

1 Die Daten von Michalik 2012 werden nachfolgend zitiert als Mi, die Daten von Haller als Ha). 
ebenfalls nach dem Verfahren der induktiven Inhaltsanalyse nach Mayring kodiert und nach thematischen Schwerpunkten kategorisiert.

\section{Erfahrungen der Lehrkräfte mit dem Philosophieren mit Kindern}

Die Themen, die im Rahmen der Interviews mit den Lehrkräften entwickelt wurden, sind so vielfältig, dass hier nur ein kleiner Ausschnitt näher beleuchtet werden kann. Es handelt sich um die Ausführungen der Lehrkräfte zur Frage, ob sie Entwicklungen oder Veränderungen bei den Kindern oder sich selbst wahrgenommen haben, die sich ihrer Meinung nach auf das regelmäßige gemeinsame Philosophieren zurückführen lassen. Es lassen sich im Hinblick auf diesen Schwerpunkt aus den Antworten der Lehrerinnen die folgenden, in nahezu allen Interviews vorhandenen Themen rekonstruieren.

\section{Veränderung der gegenseitigen Wahrnehmung von Kindern und Lehrkräften}

Viele Lehrerinnen berichten, dass das Philosophieren zu einer veränderten Wahrnehmung und auch einer veränderten Haltung gegenüber den Kindern geführt habe. Überraschung und Erstaunen über die Denkfähigkeiten und die Tiefe der Fragen und Gedanken der Kinder werden oft als Auslöser eines veränderten Blicks beschrieben: „Ich war sehr erstaunt darüber, mit welcher Ernsthaftigkeit und Tiefe die Kinder über existenzielle Fragen nachdenken konnten“( $\mathrm{Mi}, \mathrm{E} 17)$. „Es hat mich überrascht, als ich dann mitbekam, dass die Kinder ganz, ganz viel zu sagen haben, ganz tiefsinnig eigentlich schon sich Gedanken machen, über viele Dinge schon nachdenken können, auch gemeinsam“ (Mi, C38). „Also man staunt, wie viel die diese Welt begriffen haben schon. Man ist also überrascht, wie die Sachen deuten." (Ha, AVI, 50).

Der veränderte Blick auf Fähigkeiten und Potentiale der Kinder geht einher mit einer neuen Wertschätzung und einem verstärkten Interesse an den Gedanken der Kinder, die ,viel freier denken, wenn sie sich denn dazu trauen und die Möglichkeit haben, als wir Erwachsenen“" (Ha, AIX, 34). Eine Lehrerin beschreibt, dass sie „sehr neugierig“ sei ,auf das, was die Kinder treibt, was die Kinder antreibt, was die Kinder wissen, was die Kinder für ein Bild von der Welt haben. [...] Dass sie noch mal in andere Richtungen denken. Und davon profitieren nicht nur die Kinder, sondern ich profitiere davon auch ganz ganz stark“ (Ha, AV, 8).

Neben der veränderten Wahrnehmung kindlicher Denkfähigkeiten im Allgemeinen wird häufig auch eine Erweiterung der Wahrnehmung und Revision 
von Urteilen bezogen auf einzelne Kinder beschrieben, die im philosophischen Gespräch ganz anders in Erscheinung treten als im herkömmlichen Unterricht. Dies gilt oft für Kinder, die ansonsten still und eher zurückhaltend sind: „Und das finde ich immer erstaunlich, wie schnell da Kinder, die ansonsten sehr still sind, [...] bei diesen philosophischen oder Nachdenkgesprächen sich beteiligen" (Mi, C100, A84ff.). Dies gilt auch für Kinder, die als verhaltensauffällig gelten oder von den Lernergebnissen zu den eher schwachen Schüler/innen zählen: „Es sind ganz unterschiedliche Kinder, die da anbeißen beim Philosophieren, die haben ansonsten beim Lesen, Schreiben, Rechnen große Schwierigkeiten. Das sind andere Stärken, die dann da rauskommen“ (Mi, C154). „Kinder, die verhaltensauffällig waren, haben sich wahnsinnig toll in solche Gespräche eingebracht" (Mi, E19).

Das gemeinsame Philosophieren führt nicht nur zu einer veränderten Wahrnehmung der Kinder durch die Lehrerinnen, sondern auch die Lehrkräfte fühlen sich von den Kindern anders wahrgenommen und wertgeschätzt, und das hat ,mit der Haltung zu tun, die man dem Kind in solchen Stunden entgegenbringt, als Erwachsener" (Ha, AVI, 28). Ein weiterer Grund dürfte der von vielen Lehrkräften beschriebene intensivere Kontakt zu den Kindern auch auf der zwischenmenschlichen Ebene sein, der sich durch regelmäßiges gemeinsames Philosophieren ergibt und sich auch auf den Unterricht auswirkt.

\section{Auswirkungen auf das pädagogische Handeln und die Rolle als Lehrkraft}

Die von vielen Lehrkräften beschriebene veränderte Wahrnehmung gegenüber den Denkfähigkeiten und Gedanken der Kinder hat Konsequenzen für das pädagogische Handeln und auch das professionelle Selbstverständnis: „Ich habe eine ganz andere Haltung in Gesprächssituationen auch mit kleinen Kindern wie vor zehn Jahren. Weil ich einfach wertschätzender dem Gedankengang des Kindes gegenüber stehe" (Ha, AIV, 27). Andere Lehrerinnen berichten, dass sie aufmerksames Zuhören und vor allem Zurückhaltung gelernt hätten: „Ich musste ja lernen, mich zurück zu halten, und das war für mich ein ganz wichtiger Schritt, dass ich nicht dauernd das Gespräch leite und führe. [...] Und das musste ich ganz stark lernen, das habe ich nicht gekonnt“" (Mi, B33).

Eine solche Haltung findet ihren Ausdruck auch in einer veränderten Unterrichtsgestaltung, indem Fragen und Interessen der Kindern auch außerhalb des Philosophierens verstärkt für die Planung von Unterricht aufgegriffen werden: „Wenn man früher das Thema Pubertät vielleicht so angegangen wäre, dass man mit den Kindern inhaltlich da ein Gespräch abgesteckt hätte, fragt man jetzt eher andersherum: Was interessiert euch? Was gehen euch für Gedanken durch den Kopf zu dem Thema? Also man arbeitet mehr so im Hinblick auf Fragen und Interessen der Kinder“ (Mi, A247ff.). „Als ich in der Lerngruppe das erste Mal so eine Fragenbox aufgestellt habe, da war ich fassungslos, was für tolle Fragen formuliert wurden. Und 
das war für mich eigentlich der Moment zu sehen, du musst hier gar nicht mit zig vorbereiteten Fragen in den Unterricht kommen [...], du kannst Fragen diskutieren, die von den Kindern selbst kommen und das ist dann eben dieses selbstverantwortete, selbstbestimmte, individualisierte Lernen“ (Mi, D138).

Regelmäßiges gemeinsames Philosophieren von Lehrkräften und Kindern hat auch das Potential, die eigene Rolle und das eigene Selbstverständnis als Lehrkraft zu transformieren im Sinne einer Verminderung des Machtgefälles zwischen Kindern und Lehrkraft. Was die Lehrerinnen am Philosophieren besonders schätzen, ist die Tiefe und Intensität der Gespräche und das authentische Miteinandersprechen ,auf Augenhöhe“ (Mi, B33), bei dem die klassische Rolle der Lehrerin als Vermittlerin von Wissen ausgesetzt ist: „Es ist ja auch natürlich ein anderes Lehrerbild, was entsteht. [...] Weil man auch selbst aus dieser Antwortgeberrolle herauskommt [...] und letztendlich dann wie die Kinder aber auch aushalten muss, dass man selber die Antwort nicht gibt [...]“ (Ha, AIX, 19). ,Es kommen ja auch immer wieder Überraschungen, dass sich eine Gruppe für eine Fragestellung interessiert, mit denen ich mich dann selbst auch noch einmal neu befassen muss, [...] und auf diese Frage weiß man keine Antwort. Da denkt man mit den Kindern mit und nimmt wieder diese kindliche, versucht zumindest diese kindliche Haltung anzunehmen. Und denen genauso zu folgen, wie sie versuchen, mir zu folgen" (Ha, AX, 44). Andere Lehrerinnen sprechen von der philosophischen Gesprächsgemeinschaft als einer "gleichberechtigten Truppe“ (Ha, AVIII, 8) oder einer neuen Rolle der Lehrerin als Begleiterin des Lernens der Kinder (Ha, AVI, 19).

- Solche Äußerungen deuten auf Transformationsprozesse in den Beziehungen zwischen Lehrkraft und Kindern hin, die teilweise jedoch auch mit Unsicherheiten bei den Lehrkräften verbunden sind, die sie selbst erfahren haben oder auch im Kollegium vermuten. So ist ein immer wiederkehrender Topos der Umgang mit Ungewissheit. Es handelt sich hier um Ungewissheit in mehrfacher Hinsicht, nämlich Ungewissheit im Hinblick auf

- die philosophischen Inhalte bzw. die verschiedenen Antwortmöglichkeiten auf philosophische Fragen

- die Offenheit philosophischen Fragens und Forschens überhaupt und

- den nicht planbaren Verlauf philosophischer Gespräche und den damit verbundenen Kontrollverlust.

„Man muss natürlich gut vorbereitet sein, um so ein offenes Gespräch führen zu können. Ja und das ist ja eine Form von Unsicherheit, in die man sich selber begibt. Man weiß ja nicht, worauf wird diese Stunde eigentlich hinauslaufen“ (Ha, AVI, 52f.). „Aber das ist ja eben das Gefühl des Lehrers, dass er immer so alles unter Kontrolle haben möchte. Das man nicht alles lenken kann, das muss man erst mal so akzeptieren und sich, ja, darauf einlassen. Die Fragen kommen dann ja von den Kindern" (Mi, C15). „Es gibt ja so manchmal den Einwand, dass es schwierig ist, mit Kindern im 
Unterricht zu philosophieren, weil man als Lehrende in einer Rolle ist, weil man eben auch nicht alles weiß" (Mi, E14).

Von den meisten Lehrkräfte wurden diese Elemente von Ungewissheit jedoch nicht als Problem, sondern als ein konstitutives und positives Merkmal des Philosophierens gesehen: „Man muss nicht interessiert sein an Ergebnissen, sondern wirklich auch sich darauf einlassen können, dass es vielleicht auch Richtungen einnimmt, die man sich selber so vielleicht gar nicht vorgestellt hat und dann kann es aber auch sehr bereichernd sein." (Ha, VIII, 41). Zum Beispiel, indem durch die Offenheit des Gespräches von den Kindern selbst Vernetzungen mit anderen Unterrichtsfächern oder Erfahrungen aus der eigenen Lebenswelt hergestellt werden können: „Die sortieren eben ihre Erfahrungen nicht in Fächern, sondern können dann halt flexibler zugreifen" (Mi, E17). Dass die Offenheit des philosophischen Fragens und Forschens mit den Kindern überwiegend nicht als Problem, sondern als Vorteil empfunden wird, zeigen auch die Aussagen der Lehrerinnen zur Bedeutung des Philosophierens mit Kindern für ihr eigenes Leben und ihre eigene berufliche und persönliche Entwicklung.

\section{Eigene Lerneffekte und Persönliche Bedeutsamkeit}

Die große Mehrheit der Lehrerinnen betrachtet das Philosophieren mit Kindern als eine große persönliche und berufliche Bereicherung. Was die Lehrerinnen besonders hervorheben ist immer wieder die Erweiterung des eigenen Horizontes und die Veränderung des eigenen Denkens, ausgelöst sowohl durch die Ansprüche des Philosophierens an sich als auch speziell durch den Austausch mit den Kindern und deren Sicht auf die Welt: „Für mich persönlich ist es immer ein großes Geschenk, Philosophieren mit Kindern zu unterrichten, weil ich die Welt immer wieder auch aus Kinderaugen und Kindersicht widergespiegelt bekomme. Und ich werde immer wieder an das Wesentliche im Leben erinnert. [...] Was sind die grundlegenden Fragen des Lebens?“ (Ha, AVI, 35). „Ich denke über Dinge nach, über die ich vorher nicht nachgedacht habe. [...] Ich interessiere mich auch auf eine ganz andere Art für Dinge. Also mein Blick ist echt geweitet" (Ha, AV, 8). Das Philosophieren ,stärkt die eigene Wahrnehmung von sich selbst, also sich selbst bewusst zu werden über Einstellungen zu Fragen der Gesellschaft“ (Mi, C39). „Das Philosophieren bereichert mein Leben und meine Berufstätigkeit auf eine neue Art. [...] Dass das irgendwie so eine Oase im Schulleben ist " (Ha, AVI, 19, 28).

Im Hinblick auf beobachtete Wirkungen des Philosophierens oder Veränderungen bei den Kindern stimmen die von den Lehrkräften gemachten Erfahrungen weitgehend mit den aus anderen Forschungszusammenhängen gewonnen Ergebnissen (vgl. Michalik, Wirkungen in diesem Band) überein. Genannt werden immer wieder folgende Wirkungen:

- eine Verbesserung der Kommunikationsfähigkeit und des Gesprächsverhalten auch in Konfliktsituationen 
- generell ein respektvolleres Verhalten der Kinder untereinander

- eine Verbesserung der Reflexionsfähigkeit der Kinder, die auch in anderen Unterrichtssituationen zum Beispiel in Mathematik in Form einer veränderten Herangehensweise an Aufgabenstellungen deutlich wird

- eine kritischere Haltung und ein verändertes Frageverhalten

- eine Stärkung des Selbstvertrauens der Kinder und ihrer Selbstwahrnehmung als Lernende.

Darüber hinaus wird in den Interviews jedoch auch deutlich, dass manche Lehrkräfte der Meinung sind, nicht alle Kinder mit dem Philosophieren erreichen zu können, dass das Philosophieren auch anstrengend für Kinder sein kann, und dass es auch Kinder gibt, die sich für philosophisches Fragen und Nachdenken nicht interessieren oder nicht dazu in der Lage sind, sich darauf einzulassen. Dieser Aspekt ist interessant und wichtig und sollte bei zukünftigen Forschungsvorhaben Berücksichtigung finden.

Die Ergebnisse der Interviews mit Lehrkräften stimmen weitgehend mit bereits vorliegenden Forschungsbefunden überein. Sie weisen darauf hin, dass das Philosophieren mit Kindern auf Seiten der Lehrkräfte zu einer Veränderung der Wahrnehmung und infolgedessen auch Haltung gegenüber den Kindern führen kann, die sich auf ihr pädagogisches Handeln auch in anderen Unterrichtssituationen auswirkt. Als Auslöser oder Motor der Veränderung werden immer wieder „Überraschung“ und „Erstaunen“ (Gorard/Siddiqui/See 2015: 27; Topping/Trickey 2007: 29; Pfeiffer 2012) über die Gedanken und Denkfähigkeiten der Kinder genannt. Scholl, Nichols und Burgh, die in Australien Lehrer/innen zu Auswirkungen des Philosophierens auf den eigenen Unterricht interviewt haben, sprechen hier von "Dissonanzerfahrungen“" (Scholl/Nichols/Burgh 2016: 437), die zu einer Reflexion des bisherigen Selbstverständnisses und der eigenen Rolle als Lehrkraft führen. Diese wirken sich aus in einer Veränderung bisheriger Beziehungs- und Interaktionsmuster im Unterricht aus, die gekennzeichnet sind durch mehr Raum für Fragen und Interessen der Kinder, mehr Autonomie und weniger Kontrolle beim Lernen, ein stärker kollaborativ, interaktiv und dialogisch ausgerichtetes und insgesamt demokratischeres Unterrichten (Scholl 2014: 93ff., 96ff.; Gorard/Siddiqui/See 2015: 28; Siddiqui/Gorard/See 2017: 31; Scholl/Nichols/Burgh 2014: 246; Barrow 2014: 6). Solche Veränderungen im Unterricht gehen auch einher mit einer Verbesserung der Beziehungen zu den Schüler/innen und einem besseren Verstehen der Schüler/innen und deren Perspektiven auf Welt (Jenkins 1986: 36; Gorard/Sidiqui/See 2015: 27; Siddiqui/Gorard/See 2017: 31; Scholl 2014: 100; Pfeiffer 2013: 654).

Scholl, Nichols und Burgh bezeichnen die beschriebenen Veränderungsprozesse als „Pädagogische Transformation“ bei den Lehrkräften, die auf einer verbesserten Reflexionsfähigkeit bezogen auf das eigene pädagogische Handeln beruht (Scholl 2014, 89 u. 93), und die sie maßgeblich auf den Prozess 
des gemeinsamen Philosophierens zurückführen: Als Teil der philosophischen Gesprächsgemeinschaft profitieren auch die Lehrkräfte vom gemeinsamen Nachdenken und von der Originalität der Denkens der Kinder, das Lehren und Lernen wird beidseitig und es öffnet sich ein Raum für die Reflexion und Transformation von Paradigmen (ebd.: 100; Scholl/Nichols/Burgh 2016: 439). Damit verändert sich auch die „epistemische Macht“ der Lehrkräfte (Barrow 2015: 6) im Hinblick auf gleichberechtigtere und demokratischere Umgangsund Lernformen.

Nach den Gelingensbedingungen für das Philosophieren in der Schule befragt, äußerten viele Lehrkräfte Kritik am Schulsystem. Genannt wurden unter anderem zeitliche und organisatorische Strukturen, die wenig darauf ausgerichtet seien, Kinder zum selbstständigen Denken zu befähigen oder in ihrer individuellen Entwicklung zu fördern, weil eine einheitliche Leistungsstandardisierung in den Vordergrund gerückt sei. Auch eine mangelnde Unterstützung durch Kolleg/innen und Schulleitung wurde genannt, bedingt durch die häufig anzutreffende Einstufung des Philosophierens als ein netter, aber unnützer Zeitvertreib, der nicht in den zeitlichen Rahmen des Unterrichts und die allgemeinen Anforderungen des Schulbetriebs zu integrieren sei. Die Frage der Chancen und Probleme bei der Umsetzung des Philosophierens im Unterricht kann hier nicht weiter vertieft werden, sie ist ein wichtiger Ansatzpunkt für weitere Forschungen.

\section{Erfahrungen und Einschätzungen der Kinder zum Philosophieren}

In den Gruppendiskussionen mit den Kindern lag der Schwerpunkt der Gespräche auf den Fragen, was den Kindern am Philosophieren gut oder weniger gut gefällt, welche Unterschiede sie zum übrigen Unterricht sehen, und ob sich durch das Philosophieren etwas verändert habe.

Es sind im Wesentlichen zwei Momente, die aus den Gruppendiskussionen rekonstruiert werden konnten. Was den Kindern am Philosophieren besonders gut gefällt und von anderen Lern- und Unterrichtssituationen deutlich unterscheidet, sind erstens die Inhalte und zweitens ist es der Prozess des gemeinsamen Nachdenkens, wobei das Philosophieren als eine Tätigkeit für die Kinder eindeutig im Vordergrund steht. Die große Mehrheit der Kinder äußerte sich sehr positiv über ihre Erfahrungen mit dem Philosophieren. Nur wenige Kinder gaben an, dass das Philosophieren auch manchmal langweilig werden könne, wenn man das Thema nicht so spannend fände oder das Gespräch zu lang sei. 


\section{Die Inhalte des Philosophierens}

Auf der inhaltlichen Ebene wird von den Kindern häufig genannt, dass man beim Philosophieren Neues und Spannendes über ,das Leben“" oder „,die Welt" erfahren könne. Das Philosophieren hat in den Augen der Kinder offenbar eine andere Qualität als der Fachunterricht, denn es bietet neben partikularem Fachwissen einen umfassenderen Weltzugang: „Ich weiß jetzt mehr über das Leben und die Welt, über die ganze Welt halt irgendwie auch so“ (M2: 7.40). „Dass man da auch etwas über die ganze Welt erfährt und nicht nur über Rechenaufgaben oder halt irgendwelche Sachen über Tiere, sondern auch richtig über die ganze Welt“ (M2: 8.28). „Ich komme da eigentlich ganz anders raus als ich reingegangen bin [...]. Also ich erfahre dann auch viel mehr, weil es ist ja auch sehr wichtig, dass man über die Welt was weiß, deswegen speicher" ich das auch richtig doll in meinen Gedanken" (M1: 10.16). „Also, das ist einfach größeres Allgemeinwissen danach" (K1:15.22).

Als Unterschied zum übrigen Unterricht wird auch immer wieder die Offenheit für verschiedene Themen und auch für subjektiv Bedeutsames angeführt: „Dass man da eigentlich über alles reden kann, und dann kann man dann halt alles, was einen irgendwie bewegt zum Thema sagen“" (K2: 12.05).

Das philosophische Gespräch schafft offenbar Verbindungen zur „Welt“ außerhalb des Klassenzimmers und zur eigenen Lebenswelt und Person. Dies zeigt sich auch darin, dass die Kinder das Philosophieren insbesondere als einen Raum, der das Recht zur freien Meinungsäußerung und Diskussion bietet, wahrnehmen und schätzen: „Ich finde gut, dass man frei seine eigene Meinung sagen kann“ (K2: 3.10). „Ich finde es schön, dass man diskutieren kann“ (K1:18.02). „Man wird zu nichts gezwungen, was man machen muss, und man kann seine Meinung sagen" (K1:18.02). Das Philosophieren bietet die Freiheit und Gelegenheit, eigene Vorstellungen und Positionen einzubringen, was für die Kinder auch einer der wichtigsten Unterschiede zum üblichen Unterricht ist.

Ein besonders positiver Aspekt ist für die Kinder, dass das philosophische Gespräch ergebnisoffen ist, weil es keine eindeutigen Antworten gibt: „Ich finde es gut, dass keine Meinung falsch ist, aber auch keine Meinung richtig ist. Also es kann eigentlich so sein, aber es kann auch anders sein“ (B1: 2.04). „Ich finde es gut, dass es kein richtig oder falsch gibt" (K2: 14.48). Die Offenheit und Ungewissheit der philosophischen Fragen und Gesprächsthemen wird von den Kindern mehrheitlich nicht als Problem, sondern als ein besonders positiver Aspekt des Philosophierens herausgestellt, nämlich als eine Herausforderung für das eigene Denken und die Entwicklung eigener Meinungen und Standpunkte im Prozess des gemeinsamen Nachdenkens. 


\section{Der Prozess des gemeinsamen Philosophierens}

Noch deutlich häufiger als die Inhalte wird von den Kindern die Form des philosophischen Gespräches immer wieder genannt und von anderen üblichen Interaktionsmustern im Unterricht abgegrenzt: „Das ist so ein richtiges Gespräch und nicht so, einer meldet sich, sagt was, dann sagt die Lehrerin was, dann meldet sich der Nächste, sagt wieder was - das ist ganz schön“ (K1: 3.35). „Beim Philosophieren ist es auch nicht wie sonst im Unterricht, das halt immer einer meldet sich und redet dann und dann sagt die Lehrerin was und dann arbeiten alle. Dann kann man auch mal eigenständig was dazu sagen, das ist alles offener" (K1:3.26).

\section{Austausch von Meinungen und Gedanken}

Was das Philosophieren besonders attraktiv macht, ist nicht nur die Möglichkeit, eigene Meinungen zu vertreten, sondern es ist vor allem der Austausch von Meinungen und Gedanken und das gemeinsame Aushandeln von Sinn und Bedeutungen: „Ich finde, man lernt etwas über die anderen auch, aber auch für einen selbst, man lernt die anderen so kennen, dass man sich gut vorstellen kann, was die so denken“ (K1: 11,9). „Wenn man philosophiert, weiß man auch, was die anderen denken und man kann sie besser verstehen“ (K1: 10.16).

\section{Herausforderungen für das eigene Denken}

Mehr noch als das gegenseitige Kennenlernen zeichnet sich das philosophische Gespräch als Herausforderung für das eigene Denken und eigene Positionen aus. Die Kinder betrachten das gemeinsame Philosophieren auch explizit als eine Möglichkeit, das eigene Denken zu erweitern oder zu verändern: „Ich finde das auch gut, weil man sich von anderen Kindern beeinflussen lässt und dann über seine Meinung nachdenkt, die dann vielleicht doch nicht ganz richtig ist, weil andere dann vielleicht noch was sagen, wo man nochmal nachdenken drüber kann" (K2: 1.10). „Also, ich finde es auch sehr gut, wenn jemand sagt, das ist richtig und der andere sagt, na ja, vielleicht nicht. Dann muss man ja wieder nachdenken. Das mag ich oder finde es gut“ (B1: 3.06). „Man ändert auch jedes Mal, wenn man über etwas spricht, seine Meinung darüber, nicht immer komplett, aber man denkt trotzdem drüber nach und denkt, hm, es könnte ja auch so und so sein“ (K1: 12.64). „Ich ändere auch oft meine Meinung, wenn ich die Meinungen von anderen höre und ihre Begründungen [...] aber die Begründungen sind wichtig“" (K1: 16.09).

Sehr viele Äußerungen beziehen sich auf die Offenheit des Prozesses und die Vielfalt von Meinungen und Sichtweisen im philosophischen Gespräch, wobei die Ungewissheit hinsichtlich der ,richtigen“ Antwort überwiegend als eine interessante Herausforderung zum Überdenken und Differenzieren eigener Positionen betrachtet wird. Dass Ungewissheit auch anstrengend und schwierig sein kann, wird nur vereinzelt und eher von jüngeren Kindern 
geäußert: „Mir gefällt ein bisschen nicht, dass gar nichts richtig ist, weil dann kann man nichts Richtiges sagen“ (B1: 5.05). „Wenn der eine sagt, das ist richtig und der andere meint nein, dann kann man halt nicht zwei Sachen nehmen und das ist ganz schwierig“ (B1: 6.53).

\section{Veränderung des eigenen Denkens}

Interessant sind die Aussagen der Kinder zur Frage, ob die philosophischen Gespräche etwas bei ihnen verändert hätten. Hier wird häufig eine Veränderung des eigenen Denkens konstatiert: „Also ich glaube, ich denke jetzt auch ein bisschen mehr über andere Sachen insgesamt nach“ (K2: 5.14). „Also man überlegt jetzt noch mehr, also wenn man etwas sagt, finde ich, als früher“ (K1:3.17). „Ich kann jetzt irgendwie besser nachdenken, ich weiß nicht woher das kommt, aber ich denke vom Philosophieren“ (B1: 13.49). „Wir denken ja über mehr Sachen nach, man kann genauer nachdenken über Sachen allgemein“ (K2: 3.11).

Die Gruppendiskussionen zeigen eindrucksvoll die große Bereitschaft und Fähigkeit der Kinder, sich auf den Prozess des gemeinsamen Nachdenkens einzulassen und die spannungsreiche Vielfalt von Meinungen, Perspektiven sowie die Ungewissheit im Hinblick auf die Ergebnisse philosophischen Fragens und Forschens als eine positive Erfahrung und Herausforderung für das eigene Denken und Weiterdenken zu betrachten. Der Austausch mit den anderen Kindern, die gemeinsame Suche nach Sinn und Bedeutung scheint einen produktiven Umgang mit Ungewissheit besonders zu unterstützen: „Wenn es unterschiedliche Meinungen gibt, dann überleg ich jetzt noch mal, ist das richtig? Dann sage ich das jetzt einfach mal und dann überlegt man dann halt alle zusammen" (K2: 5.14).

Verschiedene Studien, in denen Kinder zu ihren subjektiven Einschätzungen teils im Rahmen von Einzelinterviews oder auf der Basis standardisierter Fragebögen (teilweise zusätzlich mit zusätzlichen offenen Antwortmöglichkeiten) befragt wurden, bestätigen die aus den Gruppendiskussionen gewonnenen Ergebnisse. Was das Philosophieren aus der Sicht der Schüler/innen auszeichnet und besonders attraktiv macht, ist neben der inhaltlichen Dimension vor allem der Prozess des gemeinsamen Nachdenkens, es ist die Möglichkeit der freien Meinungsäußerung und es ist der Austausch verschiedener Sichtweisen und Meinungen und die damit verbundene Möglichkeit, das eigene Denken zu verändern und weiter zu entwickeln (Barrow 2015: 10ff.; Gorard/Siddiqui/See 2015: 27; Siddiqui/Gorard/See 2017: 33; Fair et al. 2015: 29; Topping/Trickey 2007: 13ff.; Pàlsson 1996: 27). Aus den Äußerungen der Kinder lässt sich das Philosophieren als eine dialogische, partizipatorische und demokratische Praxis rekonstruieren, in der die Verschiedenheit der Perspektiven und die Ungewissheit der möglichen Antworten eine besonders produktive Rolle hat. 


\section{Fazit - Transformation des Lehrens und Lernens im Modus der Ungewissheit}

Die Rekonstruktion der subjektiven Perspektive der an philosophischen Gesprächen beteiligten Akteure, der Lehrkräfte und der Kinder, macht Potentiale des Philosophierens deutlich für die Entwicklung von Lehren und Lernen, Unterricht und Schulentwicklung hin zu einem stärker dialogischen, partizipatorischen und demokratischen Miteinander.

Die Ergebnisse zeigen, dass regelmäßiges Philosophieren weitreichende Auswirkungen auf das pädagogische Selbstverständnis und unterrichtliche Handeln von Lehrkräften haben kann, im Sinne einer Transformation bisheriger Vorstellungen von Unterricht und bisheriger Umgangsweisen mit Schüler/innen. Davon ist nicht nur die professionelle Entwicklung, sondern auch die eigene Persönlichkeitsbildung der Lehrkräfte berührt. Auf Seiten der Kinder wird deutlich, dass neben den Inhalten des philosophischen Gespräches vor allem der Prozess des gemeinsamen Nachdenkens, der Austausch von Gedanken als Anlass zur Prüfung und Erweiterung eigener Positionen, besonders wichtig und bedeutsam ist.

Die für das philosophische Fragen und Forschen konstitutive Ungewissheit des Verlaufs und der Ergebnisse philosophischer Gespräche zeichnet sich dabei in den Äußerungen sowohl der Kinder als auch der Lehrkräfte als besondere Chance $\mathrm{ab}$, um Sichtweisen und Haltungen zu verändern und eigene Denkhorizonte zu erweitern. Dass dies insbesondere für die Lehrkräfte eine besondere Herausforderung darstellt, weil ein solches gemeinsames Lehren und Lernen in einem Spannungsverhältnis zur Lehrer/innenrolle, zu tradierten Vorstellungen und Formen von Unterricht steht und die von Helsper beschriebenen Antinomien des Lehrer/innenhandelns (Helsper 1996) in besonderem Maße herausfordert, mag einer der Gründe dafür sein, dass das Philosophieren mit Kindern und Jugendlichen insgesamt noch wenig Verbreitung im schulischen Leben gefunden hat.

Für zukünftige Forschung wird es interessant sein, Gelingensbedingungen des Philosophierens sowohl aus der Sicht der Kinder als auch der Lehrkräfte näher zu untersuchen und insbesondere das Moment der Ungewissheit in seinen vielfältigen Bezügen und Wirkungsweisen näher in den Blick zu nehmen.

\section{Literatur}

Barrow, Wilma (2015): „I think she's learnt how to sort of let the class speak“": an exploratory study of children's perspectives on $\mathrm{P} 4 \mathrm{C}$ as participatory pedagogy. In: Thinking skills and creativity, 17, S. 76-87. 
Fair et al. (2015): Socrates in the Schools from Scotland to Texas: Replication a study on the effects of a Philosophy for Children program. In: Journal of Philosophy in Schools, 2, 1, S. 18-37.

Flick, Uwe (2016): Qualitative Sozialforschung. Eine Einführung. 7. Auflage. Reinbek bei Hamburg: Rowohlt.

Gaparatou, Renia/Erganzaki, Marida (2015): Student's Views about Their Participation in a Philosophy Program. In: Creative Education, 6, S. 726-737

Gorard, Stephen/Siddiqui, Nadia/See, Beng Huat (2015): Philosophy for Children. Evaluation Report and Executive Summary. School of Education. Durham University.

Haller, Tamara (2015): Philosophieren mit Kindern im Fachunterricht. Eine empirische Untersuchung zu Erfahrungen und Einstellungen von Lehrerinnen und Lehrern. Masterarbeit im Studiengang Lehramt Primar- und Sekundarstufe. Universität Hamburg.

Helsper, Werner: Antinomien des Lehrerhandelns in modernisierten pädagogischen Kulturen. Paradoxe Verwendungsweisen von Autonomie und Selbstverantwortlichkeit. In: Combe, A./Helsper, W. (Hrsg.): Pädagogische Professionalität. Untersuchungen zum Typus pädagogischen Handelns. Frankfurt a. Main: Suhrkamp.

Jenkins, Joseph (1986): Philosophy for Children Programme at a Gloucestershire Comprehensive School in Great Britain. In: Thinking. The Journal of Philosophy for Children, 6, 3, S. 33-37.

Mayring, Philipp (2015): Qualitative Inhaltsanalyse. Grundlagen und Techniken. 12 , überarbeitete Auflage. Weinheim und Basel: Beltz.

Michalik, Kerstin (2012): Interviews mit Lehrkräften und Gruppendiskussionen mit Kindern zu Wahrnehmungen und Bewertungen des Philosophierens mit Kindern. Forschungsprojekt an der Fakultät für Erziehungswissenschaft der Universität Hamburg.

Pálsson, Hreinn (1996): We Think More than Before about Others and their Opinions. An Evaluation Report from Iceland. In: Thinking and Reasoning 12, S. 24-28.

Pfeiffer, Silke (2012): Philosophieren mit Kindern in Mecklenburg-Vorpommern. In: Neißer, B./Vorholt, U. (Hrsg.): Kindern philosophieren. Berlin: LIT, S. 87-114.

Pfeiffer, Silke (2013): Das Unterrichtsfach Philosophieren mit Kinder in MecklenburgVorpommern. In: Pädagogische Rundschau 67, 6, S. 651-669.

Scholl, Rosie (2014): „Inside-out Pedagogy“: Theorising Pedagogical Transformation through Teaching Philosophy. In: Australian Journal of Teaching Philosophy, 39, 6, S. 89-106.

Siddiqui, Nadia/Gorard, Stephen/See, Beng Huat (2017): Non-cognitive impacts of Philosophy for Children. School of Education Durham University.

Scholl, Rosie/Nichols, Kim/Burgh, Gilbert (2014): Transforming pedagogy through philosophical inquiry. In: International Journal of Pedagogies and Learning, 9, 3, S. 253-272.

Scholl, Rosie/Nichols, Kim/Burgh, Gilbert (2016): Connecting learning to the world beyond the classroom through collaborative philosophical inquiry. In: Asia-Pacific Journal of Teacher Education, 44, 5, S. 436-454.

Topping, Keith J./Trickey, Steve (2007): Collaborative Philosophical Enquiry for School children: Participant Evaluation at 11 Years, in: Thinking. The Journal of Philosophy for Children 18, 3, S. 23-34. 